\title{
Fast polarizers installation for ECRH and ECE in TCV
}

\author{
Miguel Silva*, Timothy Goodman, Federico Felici, Laurie Porte

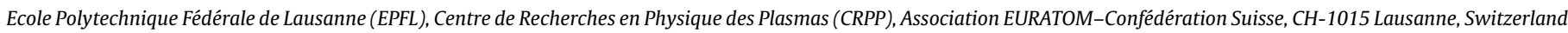

\section{A R T I C L E I N F O}

\section{Article history:}

Available online $\mathrm{xxx}$

\section{Keywords:}

TCV

Polarizer mirror

Electron cyclotron emission

Electron cyclotron heating

\begin{abstract}
A B S T R A C T
We report on the installation of fast polarizers for ECRH injection and ECE diagnostics, in the TCV tokamak. The main goal is to change the polarization during a plasma shot and react to changing conditions such as: plasma current and position, ECRH injection angles as well as ECE oblique observation angle and correlation ECE frequency. This will allow tracking of plasma variations, find optimum parameters to maximize ECRH absorption and improve the quality and consistency of ECE measurements. The polarization is varied using two fast polarizers, from General Atomics, positioned either in the evacuated transmission line for ECRH, or in a similar non-evacuated line near a real-time moveable directional antenna for ECE. A programmable driver is used to control a servo-motor and allows three operation modes during a plasma shot: fixed angle position, pre-programmed reference waveform and following of a real-time reference waveform. Preliminary tests of the motor controller achieved an $8 \mathrm{~Hz}$ bandwidth for a $\pm 4^{\circ}$ amplitude motion and a $2.2 \mathrm{~Hz}$ bandwidth for $\mathrm{a} \pm 4^{\circ}$ amplitude motion. The fast polarizers are presently in the ECE system and the control hardware is being installed.
\end{abstract}

(c) 2011 Elsevier B.V. All rights reserved.

\section{Introduction}

Electron cyclotron resonance heating (ECRH) systems are key elements in present and future magnetic confinement fusion experiments [1,2]. This versatile and powerful tool has been used for plasma heating, MHD mode suppression and fully non inductive current drive [3-5]. These systems are under continual development and further improvements are sought, e.g. real-time control [6]. In recent years, the ECRH system of the Tokamak-àConfiguration-Variable (TCV) at the CRPP/EPFL has benefited from developments in real-time feedback control of some parameters such as launcher injection angle, gyrotron power and modulation, with promising results in the control of targeted plasma instabilities [7].

The TCV ECRH system consists of two subsystems, with 6 gyrotrons at the second harmonic $(82.6 \mathrm{GHz})$ with a total power of $3 \mathrm{MW}$, and 3 gyrotrons at the third harmonic $(118 \mathrm{GHz})$, with a total power of $1.5 \mathrm{MW}$. The second harmonic (X2) system, generally used with quasi extraordinary (X)-mode power coupling, features 6 launchers for injection into TCV each with two degrees of freedom. The polarization of each beam is set by a pair of grating-polarizer mirrors housed in a matching optic unit (MOU). The mirrors are

\footnotetext{
* Corresponding author at: Ecole Polytechnique Fédérale de Lausanne (EPFL), Centre de Recherches en Physique des Plasmas (CRPP), Association EURATOM-Confédération Suisse, Station 13, CH-1015 Lausanne, Switzerland. Tel.: +41 216933498

E-mail address: miguel.silva@epfl.ch (M. Silva).
}

remote-controlled but react relatively slowly so their angles are set prior to a TCV plasma discharge where they remain during a shot. For a particular plasma discharge, the angles are calculated by the TCV ECRH control system (ECRHCS) to achieve the polarization required to maximize coupling of the chosen mode (X- or O-), accounting for the launcher angles and the plasma boundary, at one chosen shot time. As the launcher angles can be programmed to change during a shot, and the plasma parameters are continuously changing, the preset polarization will not always be optimal. In TCV, with a typical $2 \mathrm{~s}$ discharge length, the resultant fraction of stray (i.e. non-coupled) power is, in general, of little consequence, whereas in long pulsed fusion machines with more powerful ECRH systems, like ITER, such a situation might lead to damage of invessel components and/or diagnostics. To avoid this, we need to design, install and test ECRH systems for which the polarization can be varied during the plasma discharge $[8,9]$.

TCV is equipped with two electron cyclotron emission (ECE) heterodyne radiometer diagnostics [10,11], providing electron temperature measurements with $\sim 1 \mathrm{~cm}$ spatial and $10-25 \mu$ s temporal resolution. These have been used for studies of heat transport, MHD activity and fast electrons dynamics. One radiometer observes the plasma from the high field side and the other from the low field side (LFS); both observing the second harmonic. The receiving antenna used in the LFS is identical to the X2 system launchers, with 2 degrees of freedom, allowing measurements using an oblique ECE (ObECE) technique. The ECE polarization in this technique may be required to vary during a particular experiment, e.g. if the antenna is swept across the plasma cross-section and the viewing line moves 
[12]. In the present setup, the polarization has a constant value during the plasma shot and must be manually modified between shots.

The CRPP purchased a pair of fast polarizers (FP), for installation either in the ECRH system transmission line or in the front-end of the ObECE antenna. While installed in the ECRH system, they will be used for different studies, one being the testing of methods for realtime feedback control of the polarization of the injected beams. A difficulty in such a control system is to choose a reliable feedback signal that estimates the absorbed power in the intended mode $[8,9]$. The possibility of using the stray power as this signal will be investigated, for example, by studying its response to the variation of the mode purity of the injected beam (using the FP to change its polarization), as in [13]. These studies will also be used to verify the calculations of power coupling optimization. When installed in the ECE diagnostic system the FP will be used, for example, to optimize the receiver mode coupling in ObECE studies requiring fast polarization variation and for correlation ECE studies with frequency variation [12]. The ability to set remotely the polarization to any fixed value during a shot is already an improvement of the present ECE system.

This paper overviews the design, installation and tests already performed to integrate these fast polarizers into the TCV system.

\section{Design}

\subsection{System requirements}

In both the ECRH and ECE systems, the polarization required for optimum coupling to the plasma or the receiver, respectively, is determined by the linear polarization angle $\left(-90^{\circ} \leq \alpha \leq 90^{\circ}\right)$ and the elliptical polarization angle $\left(-45^{\circ} \leq \beta \leq 45^{\circ}\right)$. Any combination of these angles can be achieved with the use of two gratingpolarizer mirrors rotated about the axis normal to their surface [14]. One mirror is referred as the 'linear polarizer' and the other as the 'elliptical polarizer'. When both mirrors are placed inside the miter bend of a transmission line, the incident angle of the incoming radiation is $45^{\circ}$ so the rotation of either mirror alone changes both polarization angles [14]. The TCV ECRH system transmission line uses $\mathrm{HE}_{11}, 63.5 \mathrm{~mm}$ diameter, evacuated waveguides from General Atomics (GA), while the ObECE launcher antenna is connected to the same waveguide type (non-evacuated). Studies $[8,9,12]$ indicate that the polarizer angle requirements in some typical experiments are of the order $4-10^{\circ}$. To be able to perform experiments within TCV $2 \mathrm{~s}$ long shot, a bandwidth for polarization change between $8 \mathrm{~Hz}$ and $10 \mathrm{~Hz}$ is thus required. Taking into account ease of compatibility, (limited) market offer and required angle bandwidth the GA polarizer miter bend with fast rotation capability ( $0.1 \mathrm{~s}$ for a $90^{\circ}$ polarization rotation) was chosen. This miter bend polarizer is also foreseen for the ITER ECRH transmission line (with appropriate change of groove shape given ITER's higher frequency) [15]. So these TCV experiments will also serve as a "test bed" for the polarizers.

The FP mirror system is extremely compact, not much larger than a standard miter bend. Minimum space requirement and ease of displacement were also design specifications, since only two FP were purchased for use on the ECRH or ECE systems, as well perform laboratory tests with a low power source.

A design requirement was the ability to remotely control the FP movement, either feed-forward, (fixed angle position or a trajectory of angles) or in feed-back (using a combination of real time diagnostic measurements and control algorithms). The GA FPs are motorized with a controller that can provide these features.

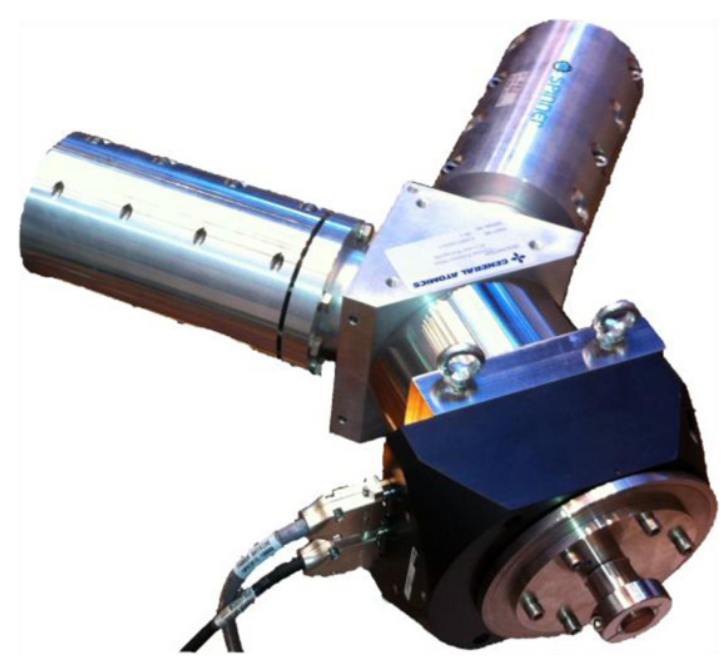

Fig. 1. GA fast polarizer (with standard GA waveguide couplers connected).

\subsection{Fast polarizers characteristics and features}

Each FP features a mirror connected via a vacuum feedthrough shaft to a direct drive servo-motor assembled into a miter bend housing (Fig. 1).

The mirrors are grooved and designed for $82.6 \mathrm{GHz}$ (gyrotron frequency). The groove profiles have a sinusoidal shape to lower the electric field peak value at the edges to avoid arcing. They may be high vacuum evacuated and will handle, in this configuration, up to $500 \mathrm{~kW}$ of EC power for short periods, since this model is not actively cooled (ohmic loss per polarizer up to $\sim 0.5 \%$ [16]). The design uses a vacuum feedthrough with a magnetic fluid seal to enhance lifetime and lower friction [17]. Specific care was taken to prevent microwave leakage by designing small clearances between the moving parts and choosing materials with low microwave absorption, e.g. for the O-ring seals. The servo-motor and its driver, from Parker [18], allow mirror accelerations of the order of $60 \mathrm{rps} / \mathrm{s}$. The servo-motor encoder has a resolution of $2^{\prime \prime}$ and the system has a typical repeatability of $\pm 6^{\prime \prime}$. The driver is programmable, in a local language, through RS232/RS485 communication and includes programmable digital and analog inputs/outputs with 12-bit/8-bit resolution, respectively. Programs, or direct commands, can control the mirror movement in two modes: "position" or "velocity". In position mode, one, or a series of, fixed angles can be defined and the mirror will move in steps, pausing at each set angle. The velocity and acceleration (positive and negative) can also be set for each stepped movement. In velocity mode, one or more velocities (in a series) can be defined together with the rotation direction and again the acceleration (positive and negative) can also be defined. In velocity mode a smoother mirror movement can be constructed without stops.

\subsection{Control system}

In line with a recent TCV control system policy for RS232 device communication, an ALIX-Geode machine will be used to communicate with the drives. Its operating system is a Linux Voyage with MdsPlus [19] installed, allowing the Geode to work as a RS232 serial server (TCP/IP based). On the client side, in the TCV central computing facilities, we use the VISTA control system featuring a live database [20]. A state-machine program, user controlled via a VISTA graphical interface, will be developed to control the FP. An MdsPlus database, with a tree-like hierarchical structure, stores all hardware and software parameters of the FP control system with the acquired data. The VISTA live database communicates 
with the MdsPlus database, through a Tree Data Interface (TDI) language process [21]. A separate TDI process communicates between the MdsPlus database and the Geode sending the user commands and program sequence (to be downloaded into the driver memory and made to run when necessary). The state-machine sets the FP for the next plasma discharge with one of the three operation modes: static mode (SM), pre-programmed mode (PPM) and realtime mode (RTM). In SM operation, a fixed polarization is requested and respective fixed polarizer angles are set using the driver's position mode. In PPM operation, the requested polarization changes in time according to a pre-shot calculation. The two calculated polarizer angle waveforms corresponding to the necessary polarization are sampled and transformed into two series of velocity points. Both drivers are set to velocity mode and a series of velocity points downloaded to each drive. In each sequence, the mirror is first moved to the (fixed) initial angle position and then the movement will be initiated by the TCV trigger signal (connected to one of the driver's digital inputs). In RTM operation, the polarizers angles are realtime feedback-controlled. Each driver is set to velocity mode and is programmed to convert a feed-back control voltage signal, sent to one of its analog inputs, to a direction and velocity. In the TCV studies using the RTM operation of the FP, polarization values are not pre-defined in the control algorithms: the polarizer mirrors are requested to rotate in specific directions at velocities proportional to the feed-back control signal value $[8,9]$.

During an experiment, the polarizer angular positions are acquired by the TCV data acquisition system. In all operational modes, the FP drivers are programmed to output the measured angle position to the analog output. This is sampled at $\sim 1 \mathrm{kHz}$ for storage. Synchronization tests, with the TCV shot cycle, are yet to be performed.

\section{Installation}

For compactness the FPs were directly connected by first connecting their integrated waveguide sections end-to-end using a standard GA coupler (split shell and a solid ring) on a precision marble surface, to ensure that the input and output waveguides lie in a plane. Next a rigid flat plate (with an integrated spirit level) was designed and attached to both miter bend housings, creating a solid FP assembly for easy of lifting.

For ECRH system, the FP assembly installation minimizes the changes to the existing transmission line by transforming a section near one of its miter bends (shown in Fig. 2). No other adjustments to the rest of the transmission line are required with the existing bellows compensating small length variations. With the existing space considerations and to facilitate access and distance to the ECE system, it is planned to modify miter bend number 2 of transmission line number 4 to install the FP assembly.

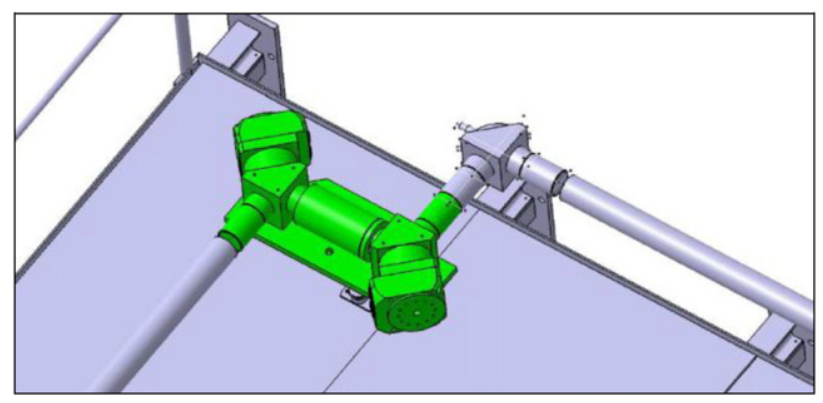

Fig. 2. Fast polarizers assembly (green) at the foreseen position of the transmission line number 4 of the ECRH system. (For interpretation of the references to color in this figure caption, the reader is referred to the web version of the article.)

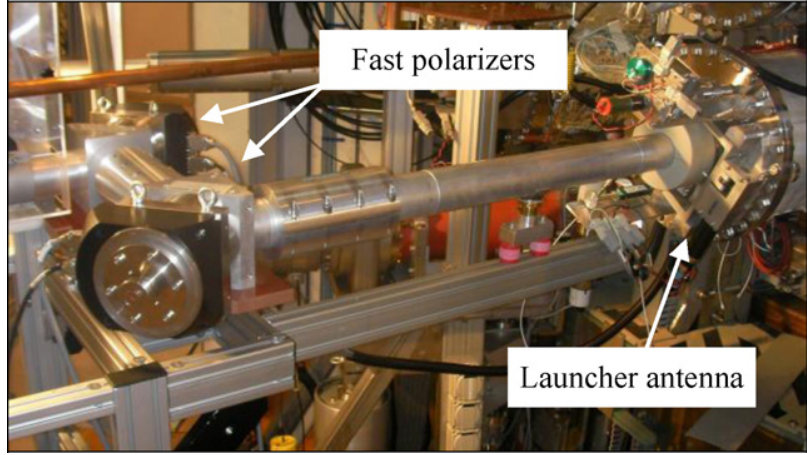

Fig. 3. The fast polarizers installed in the ECE system.

For the ECE system, the FP assembly can be installed on an existing chassis that contains a (prototype) reflectometer diagnostic system and part of the ECE diagnostic system's LFS view. This corresponds to the present situation: the chassis was modified and the FP installed, as shown in Fig. 3.

The FP rigid plate is supported at three points allowing precision height and angle adjustments. One side of the FP assembly is connected to a waveguide section from the launcher antenna (using a standard GA coupling). The other side was connected to the existing quasi-optical telescope (previously connected to the launcher antenna waveguide). The two motor drivers and the Geode were also installed in the chassis to allow the FP system to be moved into the low power test room (wheels on the chassis simplify alignment and positioning of the FP relative to the launcher antenna). In this operation the waveguide, connected to the FP, slides in an insulating adapter fixed to the back of the launcher.

When grounding the FP, for ECE or ECRH operation, care is required to avoid ground loops. Data cables were all isolated while the grounding references for power cables and mechanical parts need to be carefully chosen (as shown in Fig. 4).

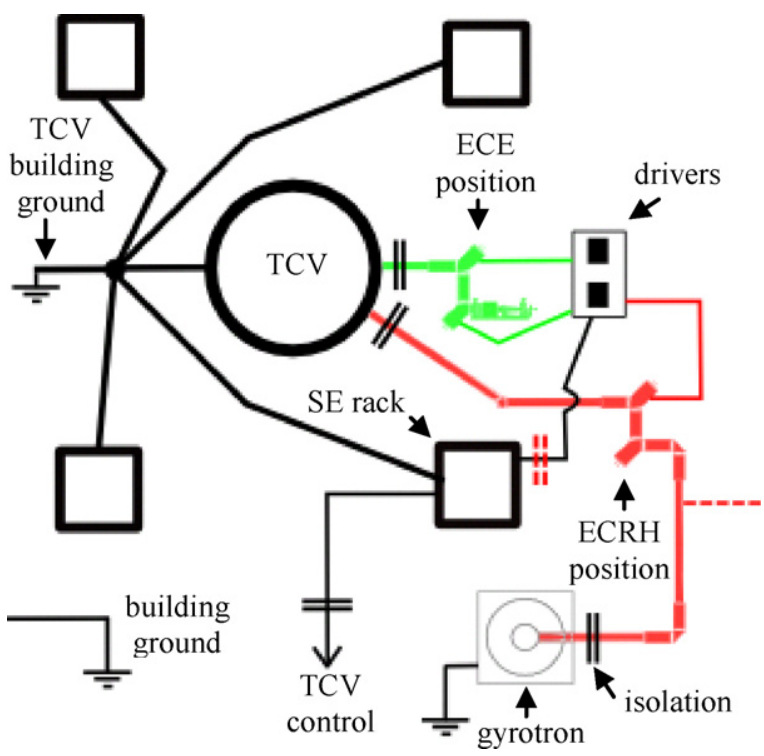

Fig. 4. General grounding scheme for the FP both in ECE (green) and ECRH positions (red). Ground isolation in different sections is represented by two small parallel lines. Dashed lines represent grounding connection/isolation when the ECRH ground is chosen to be the building ground. (For interpretation of the references to color in this figure caption, the reader is referred to the web version of the article.) 


\section{FP testing}

Initial tests measured the maximum polarizer rotation frequency of the back and forth motion for a given amplitude. A PC with Parker software was connected to the driver across a serial port. A digital oscilloscope was connected to the driver analog output that was programmed to show the instantaneous angular position. The driver was placed in position mode with acceleration set to $60 \mathrm{rps} / \mathrm{s}$ and velocity set to $5 \mathrm{rps} / \mathrm{s}$ (this value was high enough to not be a limiting factor during the tests). For $\pm 4^{\circ}$ oscillation the measured frequency was $8 \mathrm{~Hz}$ while for $\pm 40^{\circ}$ oscillation this fell to $2.2 \mathrm{~Hz}$. For these tests, the initial and final acceleration values (positive or negative) are approached smoothly (pure velocity 's-curve'). This operation is less demanding on the system's mechanics but limits the attainable frequency. A trapezoidal-like curve programming (i.e. abrupt steps in acceleration) resulted in a $10 \mathrm{~Hz}$ bandwidth for the $4^{\circ}$ amplitude motion, either in position or velocity driver mode. Further tests consisted in developing a driver program to prepare the FP for extremum seeking feedback control studies, as in [9]. PPM and RTM operations require testing to prove their viability and to optimize different parameters. These will be the next steps in our tests.

\section{Conclusions}

A decision was made to install fast polarizers in the ECRH or the ECE diagnostic systems of the TCV tokamak. System requirements were analyzed and an initial design was developed. Installation in the ECE system was performed after laboratory tests with the fast polarizers were completed. Results are in agreement with the system requirements in view of the foreseen studies. Development and tests for data acquisition as well as of system control will be carried out in the near future.

\section{Acknowledgments}

The authors are grateful to B.P. Duval, D. Fasel and J.L. Doane (GA) for their helpful discussions. This work was supported in part by the Swiss National Science Foundation.

\section{References}

[1] C. Darbos, et al., AIP Conf. Proc. 1187 (2009) 531-538.

[2] V. Erckmann, et al., AIP Conf. Proc. 691 (2003) 213-223.

[3] G. Gantenbein, et al., Phys. Rev. Lett. 85 (2000) 1242.

[4] F.A.G. Volpe, et al., Phys. Plasmas 16 (2009) 102502.

[5] O. Sauter, et al., Phys. Plasmas 8 (2001) 2199.

[6] A. Isayama, et al., Nucl. Fusion 43 (2003) 1272.

[7] J.I. Paley, et al., Plasma Phys. Control. Fusion 51 (2009) 124041.

[8] F. Felici, et al., Nucl. Fusion 50 (2010) 105003.

[9] F. Felici, et al., Rev. Sci. Instrum. 80 (2009) 013504.

[10] V.S. Udintsev, et al., Fusion Sci. Technol. 52 (2007) 161-168.

[11] T.P. Goodman, et al., Fusion Sci. Technol. 53 (2008) 196-207.

[12] T.P. Goodman, et al., AIP Conf. Proc. 1187 (2009) 551-554.

[13] D. Wagner, et al., Fusion Sci. Technol. 58 (2010) 658-665.

[14] J.L. Doane, Int. J. Infrared Millimeter Waves 13 (1992) 1727.

[15] S R.W. Callis, et al., Fusion Eng. Des. 84 (2009) 526.

[16] R.A. Olstad, et al., Proceedings of the 5th IAEA Technical Meeting on Steady State Operations of Magnetic Fusion Devices, Daejeon Republic of Korea, 2007.

[17] '63.5 mm Polarizer Miter Bends' Operating Manual, General Atomics, 2009.

[18] Parker Hannifin Corporation, 6035 Parkland Blvd, Cleveland, OH 44124, USA, Web-site: www.parker.com.

[19] G. Flor, et al., Proceedings of the 16th Symposium on Fusion Technology, London, 1990, pp. 1272-1276.

[20] Vista Control Systems, 176 Central Park Square, Los Alamos, NM87544, USA, Web-site: www.vistacontrol.com.

[21] J.A. Stillerman, et al., Rev. Sci. Instrum. 68 (1997) 939-942. 